Tatyana Maria Silva de Souza Rosendo ${ }^{1}$

Angelo Giuseppe Roncalli ${ }^{2}$

${ }^{1}$ Departamento de Saúde Coletiva, Centro de Ciências da Saúde, Universidade Federal do Rio Grande do Norte (UFRN). Av. Senador Salgado Filho 3000, Lagoa Nova. 59078-970 Natal RN Brasil. tatyana.ufrn@hotmail.com ${ }^{2}$ Departamento de Odontologia, Centro de Ciências da Saúde, UFRN.

\section{Prevalência e fatores associados ao Near Miss Materno: inquérito populacional em uma capital do Nordeste Brasileiro}

\author{
Prevalence and factors associated with Maternal Near Misses: a \\ survey of the population in a capital city of the Brazilian Northeast
}

Abstract The scope of this study was to determine the prevalence of near misses and complications during pregnancy and the puerperal period, identifying the main clinical and intervention markers and socioeconomic and demographic factors associated with near misses. It involved a cross-sectional, population-based and probabilistic study with multi-stage complex sampling design conducted in Natal, State of Rio Grande do Norte, Brazil. A validated questionnaire was given to 848 women aged 15 to 49 identified in 8,227 households in 60 census sectors. In theanal$y$ sis of associations, the Chi-square test applied and calculated the prevalence ratio $(P R)$ with Confidence Interval (CI) of $95 \%$ and 5\% significance. The prevalence of maternal near misses was 41.1/1000LB, with hospitalization in an Intensive Care Unit (19.1/1000LB) and eclampsia (13.5/1000LB) being the most important markers. The prevalence of complications during pregnancy and the puerperal period was $21.2 \%$. The highest prevalence of near misses was observed in older women, of black/brown race and low socioeconomic status. Conducting population surveys is feasible and may add important information to the study of near misses and the markers highlight the need for enhancing maternal care to reduce health inequality.

Key words Morbidity, Complications during pregnancy, Epidemiological surveys
Resumo O objetivo deste estudo foi determinar a prevalência do near miss e de complicações no período gravídico-puerperal, identificando seus principais marcadores clínicos e de intervenção e os fatores socioeconômicos e demográficos a ele associados. Trata-se de um estudo seccional, de base populacional, probabilístico, com amostra complexa, realizado em Natal/RN, Brasil. Aplicou-se um questionário validado em 848 mulheres, de 15 a 49 anos, identificadas em 8.227 domicílios de 60 setores censitários. Nas análises de associações, aplicou-se o teste Qui-quadrado e calculou-se a Razão de Prevalência (RP) com Intervalo de Confiança (IC) de 95\% e significância de 5\%. A prevalência de near miss materno foi de 41,1/1.000 $N V$, sendo a internação em UTI $(19,1 / 1.000 \mathrm{NV})$ e a eclampsia (13,5/1.000 NV) os marcadores mais referidos. A prevalência de complicações no periodo gravídico-puerperal foi de 21,2\%. A maior prevalência do near miss foi observada nas mulheres com maior idade, da raça preta/parda e com piores condições socioeconômicas. A realização de inquéritos populacionais é factível e pode acrescentar informações importantes ao estudo do near miss e seus marcadores apontam para a necessidade de fortalecimento da assistência materna para reduzir iniquidades em saúde.

Palavras-chave Morbidade, Complicações na gravidez, Inquéritos epidemiológicos 


\section{Introdução}

A Organização Mundial de Saúde relata que cerca de 289.000 mulheres morrem durante a gravidez, ou por complicações relacionadas ao parto, mesmo com os avanços científicos e tecnológicos evidenciados nas últimas décadas nas áreas de saúde materna e perinatal. A despeito do declínio de 45\% em relação aos valores dos anos 1990, percebe-se que ainda 98\% das mortes ocorrem nos países em desenvolvimento, sendo, geralmente, relacionadas a piores condições de vida e revelando as iniquidades em saúde ${ }^{1,2}$.

A morte materna é um evento trágico, não só pelas repercussões causadas no âmbito familiar, mas também porque a maioria delas é considerada evitável ${ }^{3,4}$. São óbitos evitáveis, tanto pela melhoria dos determinantes sociais através de políticas públicas que garantam os direitos humanos e reduzam as iniquidades, como pela ação dos serviços de saúde, que são os determinantes proximais na cadeia de eventos que antecedem a morte materna e têm a capacidade de atenuar as injustiças sociais, através de uma assistência universal e de qualidade ${ }^{4,5}$.

Da mesma forma que interferem na ocorrência da morte materna, os determinantes sociais e os sistemas de saúde também desempenham papel importante no contexto da morbidade materna ${ }^{2}$. O estudo dos casos mais graves de morbidade materna, também conhecidos como casos de near miss materno, tem sido colocado como estratégico para a redução da mortalidade materna e para a avaliação da assistência obstétrica $^{6}$. Recentemente, a OMS definiu um caso de near miss materno como uma mulher que quase morreu, mas sobreviveu a uma complicação que ocorreu durante a gravidez, parto ou puerpério ${ }^{7}$. A sua utilização como indicador da saúde materna apresenta algumas vantagens, como: a maior frequência de casos, a possibilidade de entrevistar a mulher sobrevivente e a possibilidade de implementar um sistema de vigilância prospectivo, por se comportar como evento sentinela ${ }^{8}$. Além disso, pode ser útil para a avaliação da qualidade do serviço de saúde e pode se comportar como um indicador do impacto de políticas públicas direcionadas à melhoria da saúde materna9 .

É possível perceber que, no contexto da morbidade materna, há um predomínio dos estudos de base hospitalar com informações obtidas a partir dos registros de prontuários ${ }^{10-13}$, embora estudos de base populacional com informações autorreferidas pelas mulheres já tenham sido realizados ${ }^{14,15}$. A necessidade de realização de inqué- ritos populacionais se justifica na medida em que eles podem ser uma alternativa para o estudo do near miss materno em locais com poucos recursos, onde as informações sobre esse evento ainda são escassas e pouco sistematizadas ${ }^{16}$.

Dessa forma, torna-se cada vez mais necessário estudar a ocorrência de complicações na gravidez - inclusive o near miss materno - e seus fatores associados na população em âmbito local, pois isso resultará num grande entendimento do processo que ocorre nessas mulheres desde o estado de saúde até o adoecimento. Além do mais, o conhecimento desta questão pode colaborar para a melhoria das políticas públicas e da assistência oferecida às mulheres que desenvolvem complicações relacionadas à gravidez, parto e puerpério, e pode contribuir para a formação de um sistema de vigilância integrado e eficaz. Portanto, o objetivo deste estudo foi determinar a prevalência do near miss materno e de complicações no período gravídico-puerperal, identificando os principais marcadores clínicos e de manejo, e os fatores socioeconômicos e demográficos relacionados ao near miss.

\section{Método}

Trata-se de um estudo seccional de base populacional realizado no município de Natal, capital do Rio Grande do Norte, Brasil. O município possui, aproximadamente, uma população de 803.739 habitantes, sendo a maior parte composta por mulheres (53\%). A taxa de fecundidade total é, em média, de 1,58 filhos por mulher em idade fértil ${ }^{17}$.

Foram incluídas no estudo mulheres em idade fértil (15-49 anos) ${ }^{18}$ residentes na zona urbana do município de Natal, e que relataram pelo menos uma gravidez nos últimos cinco anos anteriores à realização da coleta dos dados. A opção por adotar essa faixa etária, ao invés de 10-49 anos, se deu em função da possibilidade da realização de comparações internacionais e pelo fato da proporção de mulheres que engravidaram na faixa etária de 10 a 14 anos ser muito baixa.

Realizou-se um processo de amostragem probabilístico, que utilizou o desenho de amostras complexas. Inicialmente, foram sorteados os setores censitários, que foram as Unidades Primárias de Amostragem (UPA) e, em seguida, foram sorteados os domicílios que deveriam ser incluídos na pesquisa.

Considerando a sua divisão em distritos sanitários, o município de Natal foi dividido em três 
estratos (Norte, Sul/Leste e Oeste), a fim de se fazer uma distinção entre as áreas da cidade, uma vez que os distritos sanitários refletem, de certa maneira, condições socioeconômicas e de organização dos serviços de saúde. Foram sorteados 60 setores censitários distribuídos proporcionalmente nesses três estratos (22 setores no distrito Norte, 22 setores nos distritos Sul/Leste e 16 no distrito Oeste). O sorteio das Unidades Primárias de Amostragem foi realizado a partir da técnica de Probabilidade Proporcional ao Tamanho (PPT). O número de domicílios particulares permanentes urbanos (DOMPPU) foi utilizado como referência para o sorteio casual sistemático. As informações dos setores censitários foram obtidas junto ao Instituto Brasileiro de Geografia e Estatística (IBGE) tendo como base o censo de $2010^{19}$.

A amostra foi definida utilizando como referência o estudo de Souza et al. ${ }^{15}$ que encontraram, em uma pesquisa de base populacional no Brasil, a prevalência de $22 \%$ de complicações referidas no período gravídico-puerperal. Considerando esta prevalência de $22 \%$, aplicando-se uma margem de erro relativa de $15 \%$ e efeito de desenho de 1,5, o tamanho da amostra foi de 908 mulheres. Prevendo-se uma taxa de não resposta de $20 \%$, buscou-se identificar 1.135 mulheres em idade fértil ${ }^{20}$.

A definição do plano amostral seguiu os critérios estabelecidos para pesquisas de base domiciliar em países em desenvolvimento ${ }^{21}$, adaptando a técnica de percurso das quadras dos setores censitários e sorteio dos domicílios, segundo a metodologia recomendada pela OMS para inquéritos de cobertura de programas de imunização (Expanded Programme Imunization - EPI coverage survey) em países em desenvolvimento, mantendo a base probabilística do sorteio ${ }^{22}$. O número de domicílios foi determinado em função do número de tamanho da amostra, com os $20 \%$ de perda (1.135) dividido pela densidade domiciliar da população-alvo $(0,275)$, totalizando 4.128 domicílios ${ }^{23}$.

O instrumento de coleta foi um questionário estruturado, aplicado por meio de equipamentos de coleta eletrônica (tablets), que possuíam um aplicativo desenvolvido especialmente para este fim, pela equipe do Laboratório de Inovação Tecnológica em Saúde (LAIS), da Universidade Federal do Rio Grande do Norte. As questões relativas ao near miss materno e às complicações no período gravídico-puerperal foram previamente validadas por Souza et al. ${ }^{24}$.

O trabalho de campo foi realizado por uma equipe de dez entrevistadores, que foram pre- viamente capacitados quanto à utilização do instrumento de coleta e à técnica de percurso das quadras e sorteios dos domicílios. Antes da realização da pesquisa de campo, um estudo piloto foi realizado para avaliação da aplicação do instrumento e para avaliação da rotina de entrevistas nos domicílios. Foi realizada a supervisão de campo em $10 \%$ dos setores censitários da pesquisa, durante e após o término da coleta dos dados, a fim de realizar o controle de qualidade do trabalho da equipe de campo.

As variáveis dependentes do estudo são o near miss materno e as complicações ocorridas no período gravídico-puerperal. A variável near miss materno segue a mesma definição utilizada por Souza et al. ${ }^{15}$, que realizaram uma pesquisa de base populacional no Brasil e utilizaram o questionário validado em estudo anterior ${ }^{24}$. Nesse sentido, três variáveis relacionadas a intervenções (relato de histerectomia, transfusão sanguínea e admissão em UTI) e apenas uma relacionada à condição clínica (relato de eclampsia) foram as variáveis consideradas como as variáveis identificadoras de near miss materno para inquéritos populacionais ${ }^{15,24}$. A variável relativa às complicações ocorridas no período gravídico-puerperal foi composta pelo relato de qualquer complicação ocorrida nesse período. Para o cálculo da razão de prevalência de near miss materno considerou-se, no numerador, o total de casos de near miss materno e, no denominador, o número de nascidos vivos identificados no presente estudo. Para o cálculo da prevalência das complicações, dividiu-se o número de mulheres que relataram pelo menos uma das complicações pelo total de gravidezes do estudo.

As variáveis independentes correspondentes às condições socioeconômicas e demográficas foram: idade, estado civil, raça, escolaridade, renda familiar, densidade domiciliar (relação do número de pessoas no domicílio pelo número de cômodos que servem de dormitório), número de bens, ocupação (se estava trabalhando ou não no momento da entrevista) e distrito sanitário onde reside. Todos esses dados foram referentes ao momento da entrevista.

$\mathrm{Na}$ análise descritiva do near miss materno e das complicações do período gravídico-puerperal, utilizou-se a razão ou proporção com Intervalo de Confiança (IC) de 95\%. Nas análises bivariadas, as variáveis quantitativas foram categorizadas em duas categorias pelo valor da mediana e foi aplicado Qui-quadrado de Pearson para o teste de hipóteses, com limite de significância de 5\% e IC95\%. Em todas as análises foram utiliza- 
dos os requisitos de amostras complexas, incluindo os pesos e efeitos de delineamento.

A pesquisa seguiu os critérios e exigências estabelecidos pela Resolução no466/2012 ${ }^{25}$ do Conselho Nacional de Saúde (CNS) e obedeceu às recomendações do Comitê de Ética em Pesquisa do Hospital Universitário Onofre Lopes (CEP-HUOL), da Universidade Federal do Rio Grande do Norte (UFRN), ao qual o projeto foi submetido e aprovado.

\section{Resultados}

Foram entrevistadas 848 mulheres em 8.227 domicílios percorridos. Após a realização do piloto, decidiu-se por aumentar para 8.500 o número de domicílios a serem percorridos, pois se constatou uma proporção de mulheres elegíveis menor do que a esperada. Foram identificadas $1.132 \mathrm{mu}$ lheres elegíveis, no entanto, o número de mulheres entrevistadas foi menor do que o tamanho mínimo da amostra, devido a perdas relacionadas à recusa em participar da pesquisa (65) e pelo fato de a mulher elegível não ter sido encontrada após três visitas do entrevistador (219). Como o tamanho mínimo da amostra era de 908 mulheres, a taxa de resposta foi de $93,4 \%$, ou seja, uma perda de menos de 7\%, a qual não interferiu na precisão das estimativas. Considera-se, portanto, para este estudo, que a perda amostral não introduziu erro sistemático, tendo, neste caso, efeito irrelevante sobre a significância dos resultados.

A maior parte das mulheres da amostra tinha idade entre 15 a 29 anos (56,5\%), era da raça parda $(48,9 \%)$, era casada ou possuía união estável $(68,7 \%)$, cursou até o ensino fundamental ou médio $(81,5 \%)$ e possuía renda familiar de até R\$ $1.000,00(54,9 \%)$. A maior parte delas pertencia ao distrito sanitário Norte $(40,7 \%)$ e a maioria das gravidezes ocorreu entre os anos de 2010 a 2012 (64,9\%). Houve apenas 2,4\% de gestação múltipla e apenas $0,4 \%$ de bebês natimortos. A Tabela 1 apresenta a caracterização da amostra.

De acordo com a definição adotada pelo estudo, a razão de near miss materno foi de 41,1/1.000 $\mathrm{NV}$, destacando-se a internação em UTI como o critério identificador mais autorreferido, com uma razão de 19,1/1.000 NV. A Tabela 2 apresenta as razões segundo os critérios definidores de near miss materno.

A prevalência de complicações no período gravídico-puerperal, considerando as condições clínicas e intervenções, foi de 21,2\% (Tabela $3)$. A hemorragia $(10,7 \%)$ e a infecção urinária
Tabela 1. Caracterização da amostra. Natal, RN, 2014.

\begin{tabular}{lrrr}
\hline Características das mulheres $^{*}$ & $\mathbf{n}^{* *}$ & \% $^{* * *}$ & \multicolumn{1}{c}{ IC95\% } \\
\hline Idade & & & \\
$15-29$ anos & 480 & 56,5 & $52,4-60,5$ \\
$30-39$ anos & 301 & 35,5 & $32,0-39,0$ \\
$40-49$ anos & 64 & 8,1 & $6,3-10,3$ \\
Total & 845 & 100 & \\
Raça & & & \\
Branca & 286 & 33,1 & $28,6-37,9$ \\
Negra & 115 & 13,3 & $9,5-18,3$ \\
Parda & 410 & 48,9 & $43,8-54,0$ \\
Amarela & 28 & 3,5 & $2,4-5,1$ \\
Indígena & 9 & 1,2 & $0,6-2,7$ \\
Total & 848 & 100 & \\
Estado civil & & \\
Casada & 260 & 34,3 & $29,2-39,7$ \\
União estável & 279 & 34,5 & $27,5-42,1$ \\
Solteira & 224 & 29,2 & $23,2-36,0$ \\
Desquitada & 5 & 0,6 & $0,3-1,4$ \\
Divorciada & 6 & 0,8 & $0,3-1,7$ \\
Viúva & 6 & 0,8 & $0,3-1,6$ \\
Total & 780 & 100 & \\
Escolat & &
\end{tabular}

Escolaridade

Nível superior

Nível médio/fundamental

Total

$153 \quad 18,5 \quad 13,7-24,6$

$\begin{array}{lll}679 & 81,5 & 75,4-86,3\end{array}$

$832 \quad 100$

Renda familiar

Acima de R $\$ 1.000,00 \quad 305 \quad 45,1 \quad 38,9-51,4$

Até $\mathrm{R} \$ 1.000,00$

Total

$39454,948,6-61,1$

$699 \quad 100$

Ano da gravidez

2012

$214 \quad 24,7 \quad 21,3-28,3$

2011

$16920,0 \quad 17,1-23,4$

$171 \quad 20,2 \quad 17,3-23,4$

$118 \quad 14,2 \quad 11,8-17,1$

$\begin{array}{lll}99 & 11,7 & 9,8-13,9\end{array}$

$\begin{array}{lll}77 & 9,2 & 7,1-11,8\end{array}$ $848 \quad 100$

Total

Gestação múltipla

Sim

Não

Total

$20 \quad 2,4 \quad 1,6-3,8$

$818 \quad 97,6 \quad 96,2-98,4$

$848 \quad 100$

Nascido Vivo

Sim

Não

$80899,6 \quad 98,8-99,8$

$4 \quad 0,4 \quad 0,2-1,2$

Total

Distrito Sanitário

Norte

812100

Oeste

$345 \quad 40,7 \quad 27,5-55,5$

Sul/Leste

$324 \quad 37,3 \quad 23,9-52,9$

$\begin{array}{llll}179 & 22,0 & 13,3-34,0\end{array}$

$848 \quad 100$

${ }^{*}$ Dados relativos ao momento da entrevista. ${ }^{* *}$ Dados absolutos da amostra sem os pesos e efeitos do delineamento. O total de casos é de 848. Variáveis com valor total diferente desse é consequência de dados perdidos. ${ }^{* * *}$ Proporção estimada considerando os pesos e efeitos do delineamento. 
Tabela 3. Frequência absoluta e relativa e Intervalo de Confiança (IC95\%) de complicações autorreferidas no período gravídico-puerperal. Natal, RN, 2014.

\begin{tabular}{lrrr}
\hline $\begin{array}{l}\text { Complicações (condições } \\
\text { clínicas ou intervenções) }\end{array}$ & $\mathbf{n}^{*}$ & \% $^{* *}$ & IC $\mathbf{9 5 \%}{ }^{* *}$ \\
\hline Condições clínicas & & & \\
Hemorragia & 85 & 10,7 & $8,4-13,4$ \\
$\quad$ Infecção urinária & 91 & 10,7 & $8,7-13,0$ \\
$\quad$ Sepse & 44 & 5,2 & $3,8-7,1$ \\
Icterícia & 37 & 4,5 & $3,1-6,3$ \\
Pré-eclampsia/eclampsia & 34 & 4,3 & $2,9-6,2$ \\
Hipertensão crônica & 26 & 2,8 & $1,6-4,8$ \\
Abortamento & 11 & 1,6 & $0,9-2,7$ \\
Eclampsia & 10 & 1,3 & $0,7-2,3$ \\
$\quad$ Diabetes gestacional & 6 & 0,8 & $0,3-1,8$ \\
Intervenções & & & \\
$\quad$ Permanência pós-parto & 44 & 5,4 & $3,9-7,4$ \\
> de 1 semana & & & \\
Transferência inter-hospital & 35 & 4,2 & $2,9-6,0$ \\
Internação em UTI & 16 & 1,8 & $1,1-3,1$ \\
Transfusão sanguínea & 10 & 1,2 & $0,6-2,3$ \\
$\quad$ Laparotomia & 11 & 1,1 & $0,5-2,2$ \\
Ventilação mecânica & 7 & 0,9 & $0,4-2,4$ \\
Histerectomia & 2 & 0,2 & $0,1-0,9$ \\
Alguma dessas complicações & $\mathbf{1 7 6}$ & $\mathbf{2 1 , 2}$ & $\mathbf{1 8 , 3 - 2 4 , 5}$ \\
& &
\end{tabular}

"Dados absolutos da amostra sem os pesos do delineamento. * Proporção e IC95\% estimados considerando os pesos e efeitos do delineamento. Proporção em relação ao total de gravidezes (848).

possível perceber condições desfavoráveis que apresentam maior prevalência de near miss, como: idade acima de 35 anos, ser da raça preta/ parda, ser solteira ou divorciada, ter menor escolaridade, menor renda familiar, maior densidade domiciliar e menor número de bens. No entanto, não houve diferença estatisticamente significativa para nenhuma das variáveis socioeconômicas e demográficas.

\section{Discussão}

A prevalência do near miss materno encontrada no presente estudo $(41,1 / 1.000 \mathrm{NV})$ foi maior que a encontrada por Souza et al. ${ }^{15}$ no seu estudo de base populacional $(21,1 / 1.000 \mathrm{NV})$, realizado no Brasil, adotando a mesma definição pragmática. No entanto, se considerarmos que a prevalência encontrada no presente estudo foi composta principalmente pelos critérios de manejo e/ou de intervenção, percebe-se que ela está de acordo com a variação da prevalência na literatura $(0,4-$ 45/1.000 NV), quando da adoção desses critérios identificadores dos casos de near miss materno ${ }^{26}$.

Com relação às condições marcadoras, foi possível constatar maior prevalência de internação em UTI $(19,1 / 1.000 \mathrm{NV})$ seguida de eclampsia $(13,5 / 1.000 \mathrm{NV})$, transfusão sanguínea $(11,3 / 1.000 \mathrm{NV})$ e histerectomia $(2,3 / 1.000$ $\mathrm{NV})$, enquanto Souza et al. ${ }^{15}$ encontraram maior prevalência para transfusão sanguínea $(8,1 / 1.000$ $\mathrm{NV})$, seguida de eclampsia $(6 / 1.000 \mathrm{NV})$, internação em UTI $(5,2 / 1.000 \mathrm{NV})$ e histerectomia $(2,2 / 1.000 \mathrm{NV})$. Provavelmente, as diferenças encontradas, tanto em relação à razão de near miss materno, como no que diz respeito às suas condições marcadoras, tenham acontecido em função de que os resultados do estudo de Souza et al. ${ }^{15}$ referem-se ao Brasil, sendo, portanto, uma

Tabela 2. Razão de near miss materno autorreferido por 1.000 NV. Natal, RN, 2014.

\begin{tabular}{|c|c|c|c|}
\hline $\begin{array}{l}\text { Critério identificador } \\
\text { de near miss materno }\end{array}$ & $\mathbf{n}^{*}$ & $\begin{array}{c}\text { Razão de near miss } \\
\text { materno por } 1.000 \mathrm{NV}^{* *}\end{array}$ & $\begin{array}{c}\text { IC 95\% } \\
(\text { por } 1.000 \mathrm{NV})^{* *}\end{array}$ \\
\hline Internação em UTI & 16 & 19,1 & $11,2-32,2$ \\
\hline Eclampsia & 10 & 13,5 & $7,5-24,2$ \\
\hline Transfusão sanguínea & 10 & 11,3 & $5,7-22,4$ \\
\hline Histerectomia & 2 & 2,3 & $0,5-9,8$ \\
\hline Near miss materno (algum desses critérios) & 33 & 41,1 & $28,1-59,7$ \\
\hline
\end{tabular}

“Dados absolutos da amostra sem os pesos do delineamento. ${ }^{* *}$ Razão e IC95\% estimados considerando os pesos e efeitos do delineamento. $\mathrm{O}$ total de nascidos vivos é de 808 . 
Tabela 4. Frequência absoluta e relativa e significância estatística ( $p$ ) das mulheres com e sem near miss materno segundo as variáveis socioeconômicas e demográficas. Natal, RN, 2014.

\begin{tabular}{|c|c|c|c|c|c|c|c|}
\hline \multirow{2}{*}{$\begin{array}{c}\text { Variáveis socioeconômicas } \\
\text { e demográficas }\end{array}$} & \multicolumn{2}{|c|}{$\begin{array}{c}\text { Sem } \\
\text { Near Miss }\end{array}$} & \multicolumn{2}{|c|}{$\begin{array}{c}\text { Com } \\
\text { Near Miss }\end{array}$} & \multicolumn{2}{|c|}{ Total } & \multirow[t]{2}{*}{$\mathbf{p}^{* * *}$} \\
\hline & $\mathbf{n}^{* *}$ & $\%^{* * *}$ & $\mathbf{n}^{* *}$ & $\%^{* * *}$ & $\mathbf{n}^{* *}$ & $\%^{* * *}$ & \\
\hline Idade & & & & & & & 0,178 \\
\hline Até 34 anos & 649 & 96,5 & 22 & 3,5 & 671 & 78,7 & \\
\hline 35 ou mais & 164 & 94,4 & 10 & 5,6 & 174 & 21,3 & \\
\hline Raça & & & & & & & 0,052 \\
\hline Branca & 280 & 97,9 & 6 & 2,1 & 286 & 34,7 & \\
\hline Preta/Parda & 499 & 94,9 & 26 & 5,1 & 525 & 65,3 & \\
\hline Estado civil & & & & & & & 0,386 \\
\hline Casada/União estável & 519 & 96,3 & 20 & 3,7 & 539 & 68,7 & \\
\hline Solteira/divorciada & 230 & 94,9 & 11 & 5,1 & 241 & 31,3 & \\
\hline Escolaridade & & & & & & & 0,350 \\
\hline Nível superior & 149 & 97,4 & 4 & 2,6 & 153 & 18,5 & \\
\hline Nível médio/fundamental & 651 & 95,7 & 28 & 4,3 & 679 & 81,5 & \\
\hline Renda familiar & & & & & & & 0,338 \\
\hline Acima de $\mathrm{R} \$ 1.000,00$ & 294 & 96,2 & 11 & 3,8 & 305 & 45,1 & \\
\hline Até $\mathrm{R} \$ 1.000,00$ & 374 & 94,9 & 20 & 5,1 & 394 & 54,9 & \\
\hline Ocupação & & & & & & & 0,237 \\
\hline Trabalha & 313 & 94,9 & 17 & 5,1 & 330 & 39,3 & \\
\hline Não trabalha & 502 & 96,7 & 16 & 3,3 & 518 & 60,7 & \\
\hline Densidade domiciliar (moradores/cômodos dorm) & & & & & & & 0,719 \\
\hline Até 2 mor/dorm & 452 & 96,1 & 17 & 3,9 & 469 & 57,2 & \\
\hline Acima de 2mor/dorm & 349 & 95,6 & 16 & 4,4 & 365 & 42,8 & \\
\hline Número de bens & & & & & & & 0,242 \\
\hline Acima de 5 bens & 401 & 96,8 & 13 & 3,2 & 414 & 52,1 & \\
\hline De 1 a 5 bens & 398 & 94,9 & 20 & 5,1 & 418 & 48,8 & \\
\hline Distrito Sanitário & & & & & & & 0,832 \\
\hline Sul/Leste & 170 & 95,7 & 9 & 4,3 & 179 & 22,0 & \\
\hline Norte/Oeste & 645 & 96,0 & 24 & 4,0 & 669 & 78,0 & \\
\hline
\end{tabular}

*Dados relativos ao momento da entrevista. ${ }^{* *}$ Dados absolutos da amostra sem os pesos e efeitos do delineamento. O $\mathrm{n}$ total é de 33 mulheres com near miss e de 815 mulheres sem near miss. Variáveis com soma total diferente disso indica dado perdido.

${ }^{* * *}$ Proporções estimadas considerando os pesos e efeitos do delineamento. ${ }^{* * * *}$ Valor de p referente ao teste Qui-quadrado de Pearson.

estimativa nacional que pode ter sido influenciada pelas diferenças regionais existentes em nosso país.

De toda forma, percebe-se que as doenças hipertensivas específicas da gravidez - representadas neste estudo para os casos de near miss materno apenas pelos casos de eclampsia - constituem um marcador de condição clínica relevante, corroborando com o resultado de estudos anteriores que apresentaram a eclampsia e a pré-eclampsia como as principais condições clínicas responsáveis pelos casos de near miss materno ${ }^{10,12,27,28}$.

No Brasil, aproximadamente $70 \%$ dos casos de morbidade materna grave são devido a doenças hipertensivas graves, e o índice de mortalidade devido a essas causas é de $10,7 \%{ }^{29}$. Dessa maneira, é importante a implementação de estra- tégias direcionadas às doenças hipertensivas específicas da gravidez nos diversos níveis de atenção. A identificação de casos de pré-eclampsia deve ser realizada durante o pré-natal, uma vez que é nesse momento em que a maioria desses agravos podem ser rastreados através da medida da pressão arterial e verificação de proteinúria nas consultas de rotina ${ }^{30}$. A realização de um prénatal de qualidade pode contribuir para prevenir desfechos mais graves, orientando a conduta clínica mais adequada e possibilitando o tratamento necessário com a maior brevidade ${ }^{12,27}$.

Uma vez apresentado o quadro de pré-eclampsia grave ou eclampsia, a mulher necessitará de atendimento e de recursos tecnológicos e humanos especializados, como leitos de UTI, ventilação mecânica e médicos especialistas ${ }^{30}$. 
Evidências apontam para a efetividade da utilização de sulfato de magnésio na prevenção dessas doenças, destacando a sua boa relação custo-benefício ${ }^{31,32}$. Entretanto, em estudo recente realizado no Brasil, a utilização do sulfato de magnésio se deu em menos de $70 \%$ dos casos de morbidade materna grave ${ }^{29}$.

Em relação à internação em UTI, é preciso considerar alguns pontos, como, por exemplo, o fato de ser um marcador relacionado ao manejo do paciente, diferente da eclampsia que é marcador de condição clínica. Se considerarmos os marcadores de manejo, perceberemos que em outros estudos a internação em UTI também foi um fator diretamente associado aos casos de near miss materno ${ }^{10,27}$ e está também relacionado a um pior prognóstico ${ }^{29}$. Outro ponto importante para se discutir é que a internação em UTI é consequência de uma série de condições maternas desfavoráveis, geralmente relacionadas à ocorrência de doenças hipertensivas graves como a eclampsia, e está associada a uma série de outros procedimentos de manejo ou terapêuticos, como transfusão sanguínea e/ou ventilação mecânica ${ }^{33,34}$.

Reforça-se, então, a necessidade de uma rede de assistência materna articulada, que ofereça suporte eficiente na atenção terciária, como leitos obstétricos de unidade intensiva e recursos humanos e tecnológicos capacitados ${ }^{35}$. A ineficiência na oferta de atendimento especializado, sem uma regulação da oferta em relação à demanda por leitos de UTI, é uma das principais causas responsáveis pelos atrasos, considerando o "modelo dos três atrasos" ou three delays, no atendimento às mulheres com morbidade materna grave, especialmente o "terceiro atraso", no qual, uma vez a mulher tendo conseguido chegar ao serviço de saúde, há uma demora em receber o atendimento necessário ${ }^{29,36}$.

A prevalência das complicações relacionadas ao período gravídico-puerperal foi semelhante à encontrada em outros estudos de base populacional com morbidade materna autorreferida. Souza et al. ${ }^{15}$ encontraram a prevalência de complicações na gravidez de 18\%, que variou de 15 a $22 \%$, a depender da região geográfica do Brasil. $\mathrm{Na}$ análise de inquéritos populacionais em países da América Latina, Souza et al. ${ }^{37}$ encontraram uma prevalência de $17 \%$ para o Brasil. Mais recentemente, Souza et al. ${ }^{15}$, utilizando a mesma definição pragmática que foi adotada no presente estudo, apresentaram, para o Brasil, a prevalência de $22 \%$.

Assim como no estudo de Souza et al. ${ }^{15}$, dentre as complicações relatadas, a condição clínica mais frequente foi a hemorragia e a intervenção mais autorreferida foi a permanência no hospital após o parto por mais de uma semana. A maior prevalência de hemorragia provavelmente devese ao fato de ser uma condição facilmente percebida pelas mulheres, o que não significa dizer que seja um marcador com maior acurácia em relação ao que realmente aconteceu. É preciso levar em consideração que a hemorragia não foi utilizada como marcador para near miss materno em função de sua baixa acurácia no processo de validação ${ }^{24}$.

$\mathrm{Na}$ análise de fatores socioeconômicos e demográficos associados ao near miss materno, foi possível perceber a maior prevalência de near miss materno em mulheres com maior idade, da raça preta/parda, com menor escolaridade e com piores condições socioeconômicas, assim como em outros estudos ${ }^{14,15,38-40}$. A não existência de associação estatisticamente significativa com fatores socioeconômicos e demográficos pode ser reflexo da limitação imposta pela dificuldade de distribuição do pequeno número de casos (em números absolutos) entre as categorias das variáveis.

A análise da morbidade materna, segundo as características raciais, tem sido colocada como uma das principais formas de se avaliar as iniquidades em saúde nesse campo, por ser um proxy das condições socioeconômicas, e tem sido identificada como fator associado a complicações no período gravídico puerperal em outros estudos, sendo maior a prevalência nas mulheres negras ${ }^{38}$. As diferenças na ocorrência de morbidade materna refletem o intrincado relacionamento existente entre os determinantes socioeconômicos, como a escolaridade, e as condições de saúde materna ${ }^{14,40}$. Quanto à idade, é preciso destacar que as mulheres com maior idade são consideradas de alto risco para complicações obstétricas devido a uma maior prevalência de comorbidades e/ ou multiparidade ${ }^{41}$.

Em relação às limitações do estudo, é importante colocar que, apesar de os resultados serem representativos para o município de Natal, é possível extrapolá-los para municípios ou regiões com características semelhantes. Outra limitação foi a comparação dos resultados com outros estudos, que ficou penalizada em função do critério de identificação dos casos de near miss materno adotado, por existirem poucos estudos que utilizaram a mesma metodologia ${ }^{15,16}$. A prevalência do near miss materno utilizando uma definição pragmática, praticamente composta por indicadores de manejo do paciente, pode ter sido tam- 
bém influenciada pelas características dos serviços em ofertar essas intervenções aos pacientes.

Além disso, a principal limitação deste estudo diz respeito ao fato de ter utilizado como variável dependente a morbidade autorreferida, uma vez que uma das características dessas informações é a sua possível influência pelas condições socioeconômicas e demográficas das pessoas entrevistadas ${ }^{42}$. No entanto, é preciso destacar que as pesquisas com morbidade autorreferida têm sido cada vez mais estimuladas e realizadas por instituições nacionais como o próprio Ministério da Saúde em parceria com o IBGE, como é o caso da Pesquisa Nacional de Saúde ${ }^{43}$. Apesar das suas limitações, não se pode deixar de levar em consideração que se trata de um estudo de base populacional, com dados representativos para o município, que aborda diversos aspectos não disponíveis nos sistemas de informações e que esses resultados apontam para questões importantes no contexto da assistência materna.

\section{Colaboradores}

TMSS Rosendo e AG Roncalli participaram ativamente de todas as fases do trabalho, desde sua concepção e delineamento até a redação final do artigo.

\section{Agradecimentos}

Agradecemos ao Conselho Nacional de Desenvolvimento Científico e Tecnológico (CNPq) pelo financiamento desta pesquisa.

\section{Conclusões}

A realização de inquéritos populacionais utilizando a definição pragmática de near miss é factível e pode acrescentar informações importantes relacionadas às características socioeconômicodemográficas e aos seus marcadores clínicos e de intervenção, uma vez que muitas dessas informações não estão disponíveis nos sistemas de informação, principalmente em locais onde ainda não há um sistema de vigilância desses casos.

Os marcadores clínicos e de intervenção mais prevalentes, relacionados ao near miss materno, apontam para a necessidade de fortalecimento da atenção primária, com a realização de um pré-natal de qualidade e para a importância de uma rede de saúde articulada que garanta o referenciamento e o tratamento com os recursos humanos e tecnológicos necessários na atenção terciária, reduzindo as possíveis iniquidades existentes na saúde. 


\section{Referências}

1. World Health Organization (WHO). Trends in Maternal Mortality: 1990 to 2013. Estimates by WHO, UNICEF, UNFPA, The World Bank and the United Nations Population Division. 2014 [acessado 2014 Jun 6]. Disponível em: http://apps.who.int/iris/bitstream/10665/112682/2/9789241507226_eng.pdf?ua=1

2. Graaf J, Steegers E, Bonsel G. Inequalities in perinatal and maternal health. Curr Opin Obstet Gynecol 2013; 25(2):98-108

3. Gomes FA, Márcia A, Nakano S, Almeida AM, Matuo YK. Mortalidade materna na materna perspectiva do familiar. Rev Esc Enferm USP 2006; 40(1):50-56.

4. United Nations (UN). The Millennium Development Goals Report 2013. 2013 [acessado 2014 mar 15]. Disponível em: http://www.un.org/millenniumgoals/pdf/ report-2013/mdg-report-2013-english.pdf

5. United Nations Development Programms (UNDP). A Social Determinants Approach to Maternal Health. 2011 [acessado 2013 nov 20]. Available from: http://www. undp.org/content/dam/undp/library/Democratic Governance/Discussion Paper MaternalHealth.pdf.

6. Cecatti JG, Parpinelli MA. Saúde materna no Brasil: prioridades e desafios. Cad Saude Publica 2011; 27(7):1256.

7. Say L, Souza JP, Pattinson RC. Maternal near miss-towards a standard tool for monitoring quality of maternal health care. Best Pract Res Clin Obstet Gynaecol 2009; 23(3):287-296.

8. Cecatti JG, Souza JP, Parpinelli MA, Sousa MH, Amaral E. Research on severe maternal morbidities and nearmisses in Brazil: what we have learned. Reprod Health Matters 2007; 15(30):125-133.

9. World Health Organization (WHO). Evaluating the quality of care for severe pregnancy complications The WHO near-miss approach for maternal health. 2011 [acessado 2014 mar 20]. Disponível em: http://whqlibdoc.who.int/publications/2011/9789241502221_ eng.pdf?ua $=1$

10. Souza JP, Cecatti JG, Parpinelli MA, Serruya SJ, Amaral E. Appropriate criteria for identification of near-miss maternal morbidity in tertiary care facilities: a cross sectional study. BMC Pregnancy Childbirth 2007; 7:20.

11. Lobato G, Nakamura-Pereira M, Mendes-Silva W, Dias $\mathrm{MAB}$, Reichenheim ME. Comparing different diagnostic approaches to severe maternal morbidity and nearmiss: a pilot study in a Brazilian tertiary hospital. Eur J Obstet Gynecol Reprod Biol 2013; 167(1):24-28.

12. Moraes APP, Barreto SM, Passos VM a, Golino PS, Costa JE, Vasconcelos MX. Severe maternal morbidity: a case-control study in Maranhao, Brazil. Reprod Health 2013; 10:11.

13. Pacheco AJC, Katz L, Souza ASR, Amorim MMR. Factors associated with severe maternal morbidity and near miss in the São Francisco Valley, Brazil: a retrospective, cohort study. BMC Pregnancy Childbirth 2014; 14:91.

14. Souza JP, Souza MH de, Parpinelli MA, Amaral E, Cecatti JG. Self-reported maternal morbidity and associated factors among brasilian women. Rev Assoc Med Bras 2008; 54(3):249-255.
15. Souza JP, Cecatti JG, Parpinelli M, Sousa MH, Lago TG, Pacagnella RC, Camargo RS. Maternal morbidity and near miss in the community: findings from the 2006 Brazilian demographic health survey. BJOG 2010; 117(13):1586-1592.

16. Oliveira Junior FC, Costa ML, Cecatti JG, Pinto e Silva JL, Surita FG. Maternal morbidity and near miss associated with maternal age: the innovative approach of the 2006 Brazilian demographic health survey. Clinics 2013; 68(7):922-927.

17. Programa das Nações Unidas para o Desenvolvimento (PNUD). Atlas do Desenvolvimento Humano no Brasil, 2013 [Internet]. 2013 [acessado 2013 ago 15]. Disponível em: http://atlasbrasil.org.br/2013/download

18. World Health Organization (WHO). Reproductive Health Indicators: Guidelines for their generation, interpretation and analysis for global monitoring. 2006 [acessado 2011 out 20]. Disponível em: http://whqlibdoc.who. int/publications/2006/924156315X_eng.pdf

19. Instituto Brasileiro de Geografia e Estatísitca Estatística (IBGE). Características da população e dos domicílios. Agregados por setores censitários. Resultados do Universo - Censo 2010 [Internet]. 2010 [acessado 2011 out 01]. Disponível em: http://censo2010.ibge.gov.br/resultados

20. Lwanga, SK, Lemeshow S. Sample size determination in health studies: a practical manual [Internet]. 1991 [acessado 2011 out 20]. Disponível em: http://apps.who. int/iris/bitstream/10665/40062/1/9241544058_\%28p$1-\mathrm{p} 22 \% 29 . p d f ? u a=1$

21. United Nations (UN). Household Sample Surveys in Developing and Transition Countries [Internet]. 2005. [acessado 2011 out 15]. Disponível em: https://unstats. un.org/unsd/hhsurveys/pdf/Household_surveys.pdf

22. World Health Organization (WHO). The EPI coverage survey. 2008 [acessado 2011 out 15]. Disponível em: http://whqlibdoc.who.int/hq/2008/WHO_IVB_08.07_ eng.pdf

23. Barros F, Victora C. Amostragem. In: Barros F, Victora C, organizadores. $3^{\text {a }}$ ed. Epidemiologia da saúde infantil: um manual para diagnósticos comunitários. São Paulo: Hucitec, Unicef; 1998. p. 53-78.

24. Souza JP, Cecatti JG, Pacagnella RC, Giavarotti TM, Parpinelli MA, Camargo RS, Sousa MH. Development and validation of a questionnaire to identify severe maternal morbidity in epidemiological surveys. Reprod Health 2010; 7:16.

25. Brasil. Ministério da Saúde (MS). Conselho Nacional de Saúde. Resolução no 466, de 12 de dezembro de 2012. Diário Oficial da União 2013; 13 jun.

26. Tunçalp Ö, Hindin MJ, Souza JP, Chou D, Say L. The prevalence of maternal near miss: a systematic review. BJOG 2012; 119(6):653-661.

27. Morse ML, Fonseca SC, Gottgtroy CL, Gueller E. Morbidade Materna Grave e Near Misses em Hospital de Referência. Rev Bras Epidemiol 2011; 14(2):310-322.

28. Amaral E, Souza JP, Surita F, Luz AG, Sousa MH, Cecatti JG, et al. A population-based surveillance study on severe acute maternal morbidity (near-miss) and adverse perinatal outcomes in Campinas, Brazil: the Vigimoma Project. BMC Pregnancy Childbirth 2011; 11:9. 
29. Zanette E, Parpinelli MA, Surita FG, Costa ML, Haddad SM, Sousa MH, E Silva JL, Souza JP, Cecatti JG1; Brazilian Network for Surveillance of Severe Maternal Morbidity Group. Maternal near miss and death among women with severe hypertensive disorders : a Brazilian multicenter surveillance study. Reprod Health 2014; 11(4):1-11.

30. Duley L. The Global Impact of Pre-eclampsia and Eclampsia. Semin Perinatol 2009; 33(3):130-137.

31. World Health Organization (WHO). Prevention and treatment of pre-eclampsia and eclampsia. 2011. [acessado 2014 mar 15]. Disponível em: http://whqlibdoc. who.int/publications/2011/9789241548335_eng.pdf

32. Mcdonald SD, Lutsiv O, Dzaja N, Duley L. A systematic review of maternal and infant outcomes following magnesium sulfate for pre-eclampsia / eclampsia in real-world use. Int J Gynecol Obstet 2012; 118(2):90-96.

33. Amorim MMR, Katz L, Valença M, Araújo DE. Morbidade materna grave em UTI obstétrica no recife, região Nordeste do Brasil. Rev Assoc Med Bras 2008; 54(3):261-266.

34. Lotufo A, Parpinelli MA, Haddad SM, Surita FG. Applying the new concept of maternal near-miss in an intensive care unit. Clinics 2012; 67(3):225-230.

35. Oliveira Neto AF, Parpinelli MA, Cecatti JG, Souza JP, Sousa MH. Factors associated with maternal death in women admitted to an intensive care unit with severe maternal morbidity. Int J Gynecol Obstet 2009; 105(3):261-266.

36. Thaddeus S, Maine D. Too far too walk: maternal mortality in context. Soc Sci Med 1994; 38(8):1091-1110.

37. Souza JP, Parpinelli MA, Amaral E, Cecatti G. Assistência obstétrica e complicações graves da gestação na América Latina e Caribe : análise das informações obtidas a partir de inquéritos demográficos de saúde. Rev Panam Salud Publica 2007; 21(6):396-401.

38. Creanga A a, Bateman BT, Kuklina E V, Callaghan WM. Racial and ethnic disparities in severe maternal morbidity: a multistate analysis, 2008-2010. Am J Obstet Gynecol 2014; 210(5):435.
39. Laopaiboon $\mathrm{M}$, Lumbiganon $\mathrm{P}$, Intarut $\mathrm{N}$, Mori $\mathrm{R}$, Ganchimeg T, Vogel JP, Souza JP, Gülmezoglu AM; WHO Multicountry Survey on Maternal Newborn Health Research Network. Advanced maternal age and pregnancy outcomes: a multicountry assessment. BJOG 2014; 121(Supl.):49-56.

40. Tunçalp Ö, Souza JP, Hindin MJ, Santos C a, Oliveira TH, Vogel JP, Togoobaatar G, Ha DQ, Say L, Gülmezoglu AM; WHO Multicountry Survey on Maternal and Newborn Health Research Networ. Education and severe maternal outcomes in developing countries: a multicountry cross-sectional survey. BJOG 2014; 121(Supl. 1):57-65.

41. Lamminpää R, Vehviläinen-Julkunen K, Gissler M, Heinonen S. Preeclampsia complicated by advanced maternal age: a registry-based study on primiparous women in Finland 1997-2008. BMC Pregnancy Childbirth 2012; 12:47.

42. Szwarcwald CL, Souza-júnior PRB De, Angela, Maria Pires E, Damacena GN, Viacava F. Socio-demographic determinants of self-rated health in Brazil. Cad Saude Publica 2005; 21(Supl.):54-64.

43. Szwarcwald CL, Malta DC, Pereira CA, Vieira MLFP, Conde WL, Souza Junior PRB, Damacena GN, Azevedo LO, Silva GA, Theme Filha MM, Lopes CS, Romero DE, Almeida WS, Monteiro CA. Pesquisa Nacional de Saude no Brasil: concepcão e metodologia de aplicação. Cien Saude Colet 2014; 19(2):333-342.

Artigo apresentado em 13/04/2014

Aprovado em 05/08/2014

Versão final apresentada em 13/08/2014 